PROCEEDINGS OF THE

AMERICAN MATHEMATICAL SOCIETY

Volume 138, Number 4, April 2010, Pages 1453-1459

S 0002-9939(09)10111-9

Article electronically published on December 11, 2009

\title{
Q-CURVATURE PRESCRIPTION; FORBIDDEN FUNCTIONS AND THE GJMS NULL SPACE
}

\author{
A. ROD GOVER
}

(Communicated by Matthew J. Gursky)

\begin{abstract}
On a closed even conformal manifold $(M, c)$, such that the critical GJMS operator has a non-trivial kernel, we identify and discuss the role of a finite dimensional vector space $\mathcal{N}(\mathcal{Q})$ of functions determined by the conformal structure. Using these we describe an infinite dimensional class of functions that cannot be the Q-curvature $Q^{g}$ for any $g \in c$. If certain functions arise in $\mathcal{N}(\mathcal{Q})$, then $Q^{g}$ cannot be constant for any $g \in c$.
\end{abstract}

\section{INTRODUCTION}

Branson's Q-curvature $Q^{g}$ is a natural scalar Riemannian invariant defined on even dimensional manifolds; it was introduced in the far-sighted works [9, 5, 6] of Branson-Ørsted and Branson. Other approaches to obtaining $Q^{g}$, and explicit formulae in low dimensions, may be found in [19, 20, 22. The problem of conformally prescribing Q-curvature is that of determining, on a fixed even dimensional conformal structure $\left(M^{n}, c\right)$, which functions may be the Q-curvature $Q^{g}$ for some $g \in c$; in other words, it is the problem of characterising the range of the map

$$
Q: c \rightarrow C^{\infty}(M) \quad \text { given by } \quad g \mapsto Q^{g} .
$$

This has drawn considerable attention recently (e.g. 2, 3, 12, 15, 26]), and especially the uniformisation type problem of whether one may find a metric in $c$ with $Q^{g}$ constant; see for example [13, 14, 16] and the references therein.

Throughout we shall work on an even closed (that is, compact without boundary) and connected conformal manifold $(M, c)$ of dimension $n \geq 4$. All structures will be taken to be smooth, and here $c$ is a Riemannian conformal class, i.e. an equivalence class of Riemannian metrics where $g \sim \widehat{g}$ means that $\widehat{g}=e^{2 \omega} g$ for some $\omega \in C^{\infty}(M)$. For metrics related in this way, their Q-curvatures are related by

$$
Q^{\widehat{g}}=e^{-n \omega}\left(Q^{g}+P^{g} \omega\right),
$$

where the linear differential operator $P^{g}: C^{\infty}(M) \rightarrow C^{\infty}(M)$ is the critical (meaning dimension order) GJMS operator of 21. In dimension $4, P^{g}$ is due to Paneitz. In all (even) dimensions this operator takes the form $P^{g}=\Delta^{n / 2}+$ lower order terms,

Received by the editors October 28, 2008, and, in revised form, June 10, 2009.

2010 Mathematics Subject Classification. Primary 53A30; Secondary 35J60, 53A55.

Key words and phrases. Q-curvature, curvature prescription, conformal differential geometry.

(C)2009 American Mathematical Society Reverts to public domain 28 years from publication 
where $\Delta$ indicates the Laplacian. Importantly, it is formally selfadjoint [23] and may be expressed as a composition

$$
P^{g}=\delta M d,
$$

where $\delta$ is the formal adjoint of the exterior derivative $d$, and $M$ is some other linear differential operator (from 1-form fields to 1 -form fields). It is a consequence of the Q-curvature transformation law (2) that $P^{g}$ is conformally invariant in the sense that if $g$ and $\widehat{g}$ are conformally related, as above, then $P^{\widehat{g}}=e^{-n \omega} P^{g}$. Given a linear operator $O p$, we shall write $\mathcal{N}(O p)$ for its kernel. Note that $\mathcal{N}\left(P^{g}\right)$ is conformally stable. That is, for any pair of metrics $g, \widehat{g}$ in the conformal class $c$ we have $\mathcal{N}\left(P^{g}\right)=\mathcal{N}\left(P^{\widehat{g}}\right)$. We shall usually simply write $\mathcal{N}(P)$ for this space.

Note that $\mathcal{N}(d)$ is the space of constant functions and (3) implies $\mathcal{N}(d) \subseteq \mathcal{N}(P)$. Much of the previous work on the prescription equation (2) has assumed, or restricted to settings where, the operator $P$ has trivial kernel, meaning $\mathcal{N}(d)=\mathcal{N}(P)$. However, the operator $P^{g}$ can have a non-trivial kernel. For the Paneitz operator, in dimension 4, explicit examples are due to Eastwood and Singer [17]; see also [18]. (Each of these is a conformally flat product of a sphere with a Riemann surface and admits a metric $g$ with $Q^{g}$ constant but not zero.) Thus here we restrict to $n \geq 4$ and make some first steps to study the consequences of the GJMS operator $P^{g}$ having non-trivial kernel. The first such is the existence of a class of forbidden functions, that is, functions that cannot be in the range of $Q$ (in (1)), as follows.

Theorem 1.1. On a closed connected conformal manifold $(M, c)$ with $\mathcal{N}(P) \neq$ $\mathcal{N}(d)$, there are non-zero functions $u \in \mathcal{N}(P)$ such that for no pair $(g \in c, \alpha \in \mathbb{R})$, $\alpha \neq 0$, we have $Q^{g}=\alpha u$. If $k_{Q}:=\int_{M} Q$ is not zero, or if $\operatorname{dim} \mathcal{N}(P) \geq 3$, then there are such functions $u$ which are non-constant. In any case there is a space of functions $\mathcal{F}$, with infinite dimensional linear span, such that if $f \in \mathcal{F}$, then for no pair $(g \in c, \alpha \in \mathbb{R}), \alpha \neq 0$, we have $Q^{g}=\alpha f$.

Most of the theorem is established in Section 2 via a conformally determined linear space of functions (denoted $\mathcal{N}(\mathcal{Q})$ ) as forbidden functions in $\mathcal{N}(P)$. Section 3 explores the space $\mathcal{N}(\mathcal{Q})$ and its relation to $\mathcal{N}(P)$. Proposition 3.2 gives a strengthening of Theorem 1.1 for the cases where $\int Q$ is non-zero. Theorem 3.3 shows that when $\int Q$ is not zero, $\mathcal{N}(P)$ admits a conformally invariant direct sum decomposition into the space of constant functions versus the functions suitably orthogonal to $Q$. This is also interesting because, in the case that there is $g \in c$ with $Q^{g}$ constant, it recovers the Hodge decomposition of $\mathcal{N}(P)$ with respect to that metric. The main remaining result in Section 3 is Theorem 3.4 . In the cases $\int Q \neq 0$ this shows that certain functions in $\mathcal{N}(\mathcal{Q})$ (if they arise) obstruct the prescription of constant Q-curvature.

On the sphere, forbidden functions for Q-prescription have been identified in 15 (and also for other "Q-curvatures") via Kazdan-Warner type identities; cf. 24, 4. Ostensibly, this is a different phenomenon to that discussed here.

Constructions of natural scalar Riemannian invariants with conformal transformation properties similar to the Q-curvature have been described in [19, 20, 11. Most of the results developed below extend easily to prescription problems based around such invariants.

Some issues related to studying Q-prescription when $P^{g}$ has non-trivial kernel were touched on in [25]. The current work was partly motivated by the questions 
suggested there and by discussions with Andrea Malchiodi. Conversations with Michael Eastwood are also much appreciated.

\section{The Proof of Theorem 1.1}

We recall from 7] (updated as 8]) the following observation.

Proposition 2.1. For $u \in \mathcal{N}(P), \int_{M} u Q^{g} \mu_{g}$ is conformally invariant.

Here we write $\mu_{g}$ for the canonical measure determined by the metric. For our current purposes it is useful to review the proof of the proposition.

Proof. Recall that if $\widehat{g}=e^{2 \omega} g, \omega \in C^{\infty}(M)$, then we have the Q-curvature transformation (2). Since the measure $\mu_{g}$ transforms conformally according to $\mu_{\widehat{g}}=e^{n \omega} \mu_{g}$, we have

$$
\int_{M} u Q^{\widehat{g}} \mu_{\widehat{g}}=\int_{M} u e^{-n \omega}\left(Q^{g}+P^{g} \omega\right) e^{n \omega} \mu_{g}=\int_{M} u Q^{g} \mu_{g}+\int_{M} u\left(P^{g} \omega\right) \mu_{g} .
$$

But $P^{g}$ is formally selfadjoint [23], and so the second term drops out.

Let us write $\mathcal{Q}$ for the linear operator $\mathcal{Q}: \mathcal{N}(P) \rightarrow \mathbb{R}$ given by $u \mapsto \int_{M} Q^{g} u \mu_{g}$, and $\mathcal{N}(\mathcal{Q})$ for its kernel. Proposition 2.1 may be viewed as the statement that $\mathcal{Q}$ is conformally invariant.

Lemma 2.2. If $0 \neq u \in \mathcal{N}(\mathcal{Q})$, then there is no $g \in c$ with $Q^{g}=u$.

Proof. Given $u \in \mathcal{N}(\mathcal{Q})$, suppose that for some $g^{\prime} \in c$ we have $Q^{g^{\prime}}=u$. Then $u \in \mathcal{N}(\mathcal{Q})$ implies that $0=\int_{M} u Q^{g} \mu_{g}$. Since $u \in \mathcal{N}(P)$, the right hand side is conformally invariant. So we may calculate in the metric $g^{\prime}$, whence

$$
0=\int_{M} u Q^{g^{\prime}} \mu_{g^{\prime}}=\int_{M}|u|^{2} \mu_{g^{\prime}}
$$

and so $u=0$.

Proof of the first statement in Theorem 1.1. Since $\mathcal{Q}: \mathcal{N}(P) \rightarrow \mathbb{R}$ is linear, it follows that $\mathcal{N}(\mathcal{Q})$ has codimension at most 1. Thus if $\mathcal{N}(d) \neq \mathcal{N}(P)$, then $\mathcal{N}(\mathcal{Q}) \neq\{0\}$. So the statement follows from Lemma 2.2 ,

So the non-zero elements of $\mathcal{N}(\mathcal{Q})$ are forbidden functions. In fact we should really view $\mathcal{N}(\mathcal{Q}) \backslash\{0\}$ (and more generally $\mathcal{N}(P) \backslash\{0\}$ ) as a space of constraints on the range of $Q$, as follows. If $f \in C^{\infty}(M)$ is in the range of $Q$, then it must be that there is $g \in c$ with $\int_{M} f u \mu_{g}=0$ for all $u \in \mathcal{N}(\mathcal{Q}) \backslash\{0\}$. For $g \in c$, let us write $\mathcal{N}(\mathcal{Q})^{\perp_{g}}$ for the orthogonal complement in $C^{\infty}(M)$ of $\mathcal{N}(\mathcal{Q})$ with respect to the usual $L^{2}$ inner product on $(M, g)$. Let us make the definitions

$$
\mathcal{F}^{g}:=\left\{f \in C^{\infty}(M): f \notin \mathcal{N}(\mathcal{Q})^{\perp_{g}}\right\}
$$

and $\mathcal{F}:=\bigcap_{g \in c} \mathcal{F}^{g}$. By an obvious adaption of the proof of Lemma2.2 we have the following.

Proposition 2.3. If $f \in \mathcal{F}$, then there is no pair $(g \in c, \alpha \in \mathbb{R}), \alpha \neq 0$, such that $Q^{g}=\alpha f$.

From the proof of Lemma 2.2 we also have that $\mathcal{F} \supseteq \mathcal{N}(\mathcal{Q}) \backslash\{0\}$. The last statement of Theorem 1.1 uses the following. 
Proposition 2.4. If $\mathcal{N}(\mathcal{Q})$ contains non-constant functions, then the containment $\mathcal{F} \supseteq \mathcal{N}(\mathcal{Q}) \backslash\{0\}$ is proper and the span of $\mathcal{F}$ is infinite dimensional.

Proof. Observe that if $u \in \mathcal{N}(\mathcal{Q})$, then, for example, $u^{p} \in \mathcal{F}$ for $p$ an odd positive integer. If $u$ is non-constant, then there is no linear relation among the $u^{p}$ as $p$ ranges over odd positive integers. On the other hand, $\mathcal{N}(\mathcal{Q}) \subseteq \mathcal{N}(P)$ and $\mathcal{N}(P)$ is finite dimensional since $P$ is elliptic and $M$ is compact.

In the spirit of the proof here, note that if $f: \mathbb{R} \rightarrow \mathbb{R}$ is any function with the same strict sign as the identity, then, for any $u \in \mathcal{N}(\mathcal{Q}) \backslash\{0\}, f(u)$ is in $\mathcal{F}$.

So to complete the proof of the theorem, the main remaining task is to show when $\mathcal{N}(\mathcal{Q})$ necessarily contains non-constant functions. Although various cases are easily settled, it is worthwhile to first look at the structure of $\mathcal{N}(P)$.

\section{The Structure And Role of $\mathcal{N}(P)$ AND $\mathcal{N}(\mathcal{Q})$}

Given a metric $g$ on $M$, let us write $\overline{\mathcal{Q}}^{g}: C^{\infty}(M) \rightarrow \mathbb{R}$ for the map $f \mapsto$ $\int_{M} f Q^{g} \mu_{g}$. (So $\mathcal{Q}$ agrees with the restriction of $\overline{\mathcal{Q}}^{g}$ to $\mathcal{N}(P)$.) We have the following interpretation of $\mathcal{N}(\mathcal{Q})$.

Proposition 3.1. Given a conformal class $c$ on a closed manifold $M$, we have

$$
\mathcal{N}(\mathcal{Q})=\bigcap_{g \in c} \mathcal{N}\left(\overline{\mathcal{Q}}^{g}\right) .
$$

Proof. $\subseteq$ : This is obvious from the conformal invariance of $\mathcal{Q}$.

$\supseteq$ : Since $P^{g}$ is formally selfadjoint, given $u \in C^{\infty}(M)$, it follows easily from (2) that if

$$
\int_{M} u Q^{g} \mu_{g}=\int_{M} u Q^{\widehat{g}} \mu_{\widehat{g}} \text { for all }(g, \widehat{g}) \in c \times c,
$$

then $u \in \mathcal{N}(P)$. If $u \in \bigcap_{g \in c} \mathcal{N}\left(\overline{\mathcal{Q}}^{g}\right)$, then we have the situation of the display and also that $\int_{M} u Q^{g} \mu_{g}=0$. So $u \in \mathcal{N}(\mathcal{Q})$.

Remark. Note that, since the $L^{2}$ inner product is definite, (excepting 0) the right hand side of (4) consists of functions which are manifestly not in the range of $Q$. Thus the proposition above gives an alternative proof of Lemma 2.2

For $q \in \mathbb{R}$ we use, as usual, the same notation for the constant function on $M$ with value $q$. Since $P_{k} 1=0$, it follows from Proposition 2.1 that $k_{Q}:=\int_{M} Q^{g} \mu_{g}$ is a global conformal invariant, as is well known from [9, 5. This gives immediate restrictions on the range of $Q^{g}$. Let us write $\mathcal{E}_{+}$(resp. $\mathcal{E}_{-}$) for the space of functions $f \in C^{\infty}(M)$ such that $f$ is pointwise non-negative (respectively non-positive) but not identically zero. We will write $\mathcal{E}_{ \pm}$for the union of these spaces. If $k_{Q}=0$, then we have the well known result that any $f \in \mathcal{E}_{ \pm}$is not in the range of $Q$. In fact this provides examples of $f \in \mathcal{F} \backslash(\mathcal{N}(\mathcal{Q}) \backslash\{0\})$ : when $k_{Q}=0$ we have $1 \in \mathcal{N}(\mathcal{Q})$ and for no metric $g \in c$ is $f$ orthogonal to 1. Similarly there are obvious (and well known) constraints from $1 \in \mathcal{N}(P)$ when $k_{Q} \neq 0$. For completeness we combine here these facts with our earlier results, as follows.

Proposition 3.2. If $k_{Q}=0$, then $\mathcal{F} \supseteq \mathcal{E}_{ \pm}$. In particular $\mathcal{F}$ spans an infinite dimensional vector space. If $k_{Q}>0$ (alternatively $k_{Q}<0$ ), then the functions in $\mathcal{E}_{-} \cup \mathcal{F} \cup\{0\}$ (resp. $\mathcal{E}_{+} \cup \mathcal{F} \cup\{0\}$ ) are not in the range of $Q$. 
Next we exhibit a decomposition of $\mathcal{N}(P)$ which establishes the second statement of Theorem 1.1 for the case that $k_{Q} \neq 0$. It is also of independent interest.

Theorem 3.3. Suppose that $k_{Q} \neq 0$. Then we have a conformally invariant direct decomposition

$$
\mathcal{N}(P)=\mathcal{N}(d) \oplus \mathcal{N}(\mathcal{Q})
$$

Proof of Theorem 3.3. $\mathcal{N}(P)$ and $\mathcal{N}(d)$ are conformally invariant linear subspaces of $C^{\infty}(M)$. The vector space inclusion $\mathcal{N}(d) \hookrightarrow \mathcal{N}(P)$ is split by the conformally invariant map $\mathcal{N}(P) \ni u \mapsto u_{0}:=\frac{1}{k_{Q}} \mathcal{Q}(u)$. (So explicitly (5) is given by $u=$ $u_{0}+\left(u-u_{0}\right)$ for $u \in \mathcal{N}(P)$.)

Remarks. The decomposition of the theorem is not necessarily orthogonal for any metric $g \in c$. This is linked to whether or not we may prescribe constant Qcurvature, and for $\mathcal{N}(P)$, (5) is a conformal version of the Hodge decomposition. We mean this as follows.

First suppose that there is $g \in c$ with $Q^{g}=q$ constant. Since we assume $k_{Q} \neq 0$, it follows that $q \neq 0$. By the Hodge decomposition on $(M, g)$, for any $u \in C^{\infty}(M)$ we have $u=\bar{u}+u_{1}$, where $\bar{u}$ is a constant function and $u_{1}$ is a divergence. In particular we may apply this decomposition to $u \in \mathcal{N}(P)$. We have $\bar{u} \in \mathcal{N}(d)$, $u_{1} \in \mathcal{N}(P)$ and $\int_{M} u_{1} Q^{g} \mu_{g}=q \int_{M} u_{1} \mu_{g}=0$. So $u_{1} \in \mathcal{N}(\mathcal{Q})$. On the other hand,

$$
u_{0}:=\frac{1}{k_{Q}} \int_{M} u Q^{g} \mu_{g}=\frac{q}{k_{Q}} \int_{M}\left(\bar{u}+u_{1}\right) \mu_{g}=\frac{\bar{u}}{k_{Q}} \int_{M} q \mu_{g}=\bar{u} .
$$

So we also have $u_{1}=u-u_{0}$. This shows that if $Q^{g}=q$ is constant, then, on $(M, g)$, the Hodge decomposition of $\mathcal{N}(P)$ agrees with (5). But the latter is conformally invariant and so gives a conformally invariant and canonical route to the Hodge decomposition of $\mathcal{N}(P)$, with respect to the metric $g$ that has $Q^{g}$ constant.

However, in general, we do not know there is a metric $g$ that makes $Q^{g}$ constant; there is no preferred metric to exploit for a Hodge decomposition. Nevertheless we always have the conformal decomposition (5).

Finally we note that Theorem 3.3 is closely related to the so-called "conformal harmonics" of [10] (see also [1]). In fact it gives additional structure to the $k=1$ complex of [10, Proposition 2.5].

These observations suggest the following problem. Recall that $\mathcal{E}_{ \pm}$is the space of non-zero functions which are either non-negative or non-positive.

Question 1. Suppose that $k_{Q} \neq 0$. Can the finite dimensional vector space $\mathcal{N}(\mathcal{Q})$ intersect non-trivially with $\mathcal{E}_{ \pm}$?

This question is interesting because if there are such functions, then they obstruct the prescription of constant Q-curvature. To simplify the statement, note that $f \in \mathcal{N}(\mathcal{Q}) \cap \mathcal{E}_{-}$is equivalent to $-f \in \mathcal{N}(\mathcal{Q}) \cap \mathcal{E}_{+}$.

Theorem 3.4. Assume $(M, c)$ is a closed conformal manifold with $k_{Q} \neq 0$. Suppose there is $u \in \mathcal{N}(\mathcal{Q}) \backslash\{0\}$ such that $u$ is pointwise non-negative. Then $\nexists g \in c$ with $Q^{g}$ either pointwise positive or pointwise negative. In particular there is no $g \in c$ with $Q^{g}$ constant.

Proof. From Proposition 2.1, $0=\int_{M} u Q^{g} \mu_{g}$ is conformally invariant, but for no metric is $u$ orthogonal to a strictly positive or strictly negative function. 
Note that if $k_{Q}=0$, then $\mathcal{N}(d) \subseteq \mathcal{N}(\mathcal{Q})$. So we cannot have the decomposition (5). However in this case there is the possibility that $\mathcal{N}(\mathcal{Q})=\mathcal{N}(P)$. From standard Fredholm theory, there is a characterisation of this situation, as follows.

Proposition 3.5. On a conformal manifold $(M, c)$,

$$
(\mathcal{N}(P)=\mathcal{N}(\mathcal{Q})) \Leftrightarrow\left(\exists g \in c \text { s.t. } Q^{g}=0\right) .
$$

This is simply a restatement of an observation of Malchiodi in [25, Section 3], and there the following question is suggested.

Question 2. Suppose that $k_{Q}=0$. Can there be functions in $\mathcal{N}(P)$ which are not orthogonal to $Q^{g}$ ?

As for question 1, such functions obstruct the prescription of constant $Q^{g}$.

\section{Remark and proof of Theorem 1.1}

Suppose that $k_{Q}=0$ and $\operatorname{dim} \mathcal{N}(P)=2$. Then either $\mathcal{N}(\mathcal{Q})=\mathcal{N}(P)$, and 0 is in the range of $Q$ but all other elements of $\mathcal{N}(P)$ are forbidden, or $\mathcal{N}(\mathcal{Q})=\mathcal{N}(d)$ in which case we cannot solve for constant $Q^{g}$, but we cannot identify non-constant forbidden functions in $\mathcal{N}(P)$. On the other hand, if $\operatorname{dim} \mathcal{N}(P)>2$, then it is clear that we always get non-constant (and even mixed sign) functions in $\mathcal{N}(P)$ that are not in the range of $Q$ (as claimed in Theorem 1.1). On the other hand, when $k_{Q} \neq 0$, it is immediate from, for example, Theorem 3.3 that $\mathcal{N}(\mathcal{Q}) \backslash\{0\}$ consists of non-contant functions.

\section{ACKNOWLEDGEMENTS}

The basic idea for this work arose during the conference "PDE in Conformal and Kähler Geometry", University of Science \& Technology of China, July 2008, and also the New Zealand Institute of Mathematics and its Applications (NZIMA) thematic programme "Conformal Geometry and its Applications", hosted by the Department of Mathematics of the University of Auckland in 2008. The article was written during the programme "Geometric Partial Differential Equations" at the Institute for Advanced Study, Princeton. The author is grateful for the stimulation and support of these programmes and institutions. The author is also supported by Marsden Grant No. 06-UOA-029.

\section{REFERENCES}

[1] E. Aubry and C. Guillarmou, Conformal harmonic forms, Branson-Gover operators and Dirichlet problem at infinity. J. European Math. Society, to appear, arXiv:0808.0552

[2] P. Baird, A. Fardoun, and R. Regbaoui, Q-curvature flow on 4-manifolds, Calc. Var. Partial Differential Equations 27 (2006), 75-104. MR2241307 (2007d:53107)

[3] P. Baird, A. Fardoun, and R. Regbaoui, Prescribed Q-curvature on manifolds of even dimension, J. Geom. Phys. 59 (2009), 221-233. MR2492192

[4] J.-P. Bourguignon and J.-P. Ezin, Scalar curvature functions in a conformal class of metrics and conformal transformations, Trans. Amer. Math. Soc. 301 (1987), 723-736. MR882712 (88e:53054)

[5] T.P. Branson, The functional determinant, Lecture Notes Series, 4. Seoul National University, Research Institute of Mathematics, Global Analysis Research Center, Seoul, 1993. MR.1325463 (96g:58203)

[6] T.P. Branson, Sharp inequalities, the functional determinant, and the complementary series, Trans. Amer. Math. Soc. 347 (1995), 3671-3742. MR1316845 (96e:58162) 
[7] T.P. Branson and A.R. Gover, Origins, applications and generalisations of the Q-curvature, American Institute of Mathematics, 2003, http://www.aimath.org/pastworkshops/ confstruct.html

[8] T.P. Branson and A.R. Gover, Origins, applications and generalisations of the Q-curvature, Acta Appl. Math. 102 (2008), 131-146. MR2407527

[9] T.P. Branson and B. Ørsted, Explicit functional determinants in four dimensions, Proc. Amer. Math. Soc. 113 (1991), 669-682. MR.1050018 (92b:58238)

[10] T.P. Branson and A.R. Gover, Conformally invariant operators, differential forms, cohomology and a generalisation of Q-curvature, Comm. Partial Differential Equations 30 (2005), 1611-1669. MR 2182307 (2006k:58060)

[11] T. Branson and A.R. Gover, Pontrjagin forms and invariant objects related to the Q-curvature, Commun. Contemp. Math. 9 (2007), 335-358. MR2336821 (2008h:58064)

[12] S. Brendle, Convergence of the Q-curvature flow on $S^{4}$, Adv. Math. 205 (2006), 1-32. MR2254307 (2007f:53075)

[13] S.-Y.A. Chang, M.J. Gursky, and P.C. Yang, A conformally invariant sphere theorem in four dimensions, Publ. Math. Inst. Hautes Études Sci. 98 (2003), 105-143. MR2031200 (2005b:53054)

[14] S.-Y.A. Chang and P.C. Yang, Extremal metrics of zeta function determinants on 4-manifolds, Ann. of Math. (2) 142 (1995), 171-212. MR1338677 (96e:58034)

[15] P. Delanoë and F. Robert, On the local Nirenberg problem for the Q-curvatures, Pacific J. Math. 231 (2007), 293-304. MR2346498

[16] Z. Djadli and A. Malchiodi, Existence of conformal metrics with constant Q-curvature, Ann. of Math. (2) 168 (2008), 813-858. MR2456884(2009h:53074)

[17] M.G. Eastwood and M.A. Singer, The Fröhlicher spectral sequence on a twistor space, J. Differential Geom. 38 (1993), 653-669. MR1243789 (94k:32050)

[18] M.G. Eastwood and J. Slovak, A primer on Q-curvature, American Institute of Mathematics, 2003, http://www.aimath.org/pastworkshops/confstruct.html

[19] C. Fefferman and K. Hirachi, Ambient metric construction of Q-curvature in conformal and CR geometries, Math. Res. Lett. 10 (2003), 819-831. MR2025058(2005d:53044)

[20] A.R. Gover and L.J. Peterson, Conformally invariant powers of the Laplacian, $Q$-curvature, and tractor calculus, Comm. Math. Phys. 235 (2003), 339-378. MR1969732 (2004d:58047)

[21] C.R. Graham, R. Jenne, L.J. Mason, and G.A. Sparling, Conformally invariant powers of the Laplacian. I. Existence, J. London Math. Soc. (2) 46 (1992), 557-565. MR.1190438 (94c:58226)

[22] C.R. Graham and K. Hirachi, The ambient obstruction tensor and Q-curvature, AdS/CFT correspondence: Einstein metrics and their conformal boundaries, 59-71, IRMA Lect. Math. Theor. Phys., 8, Eur. Math. Soc., Zurich, 2005. MR2160867 (2006k:53045)

[23] C.R. Graham and M. Zworski, Scattering matrix in conformal geometry, Invent. Math. 152 (2003), 89-118. MR.1965361 (2004c:58064)

[24] J.L. Kazdan and F.W. Warner, Scalar curvature and conformal deformation of Riemannian structure, J. Differential Geometry 10 (1975), 113-134. MR0365409 (51:1661)

[25] A. Malchiodi, Conformal metrics with constant Q-curvature, SIGMA Symmetry Integrability Geom. Methods Appl. 3 (2007), Paper 120. MR2366902 (2008m:53089)

[26] A. Malchiodi and M. Struwe, Q-curvature flow on $S^{4}$, J. Differential Geom. 73 (2006), 1-44. MR2217518 (2007c:53087)

Department of Mathematics, The University of Auckland, Private Bag 92019, AuckLAND 1142, New ZeALAND

E-mail address: gover@math.auckland.ac.nz 